Image Processing, Geoinformatics and Information Security

\title{
INTERPOLATION FOR HYPERSPECTRAL IMAGES COMPRESSION
}

\author{
M.V. Gashnikov \\ Samara National Research University, Samara, Russia
}

\begin{abstract}
The comparative research of the different interpolators for hierarchical compression of hyperspectral images is performed. The compression method based on a hierarchical grid interpolation is considered. Standard interpolation schemes are described and the rank interpolator is proposed as a part of this compression method. The computational experiments are performed on real images of 16-bit hyperspectrometers. The results of the interpolators' comparison are considered in the coordinates "error - compression ratio."
\end{abstract}

Keywords: interpolation, digital image compression, hyperspectral image, maximum error.

Citation: Gashnikov MV. Interpolation for hyperspectral images compression. CEUR Workshop Proceedings, 2016; 1638: 327-333. DOI: $10.18287 / 1613$ 0073-2016-1638-327-333

\section{Introduction}

Research areas related to the processing of hyperspectral images attract more and more attention in recent years [1-3]. Remote sensing data of this type are increasingly used in various applications [4-10]. However, one of the main problems that disturb the use of hyperspectral images is the size of this data. One such image includes several hundred channels of two-dimensional size of several thousands of pixels in each coordinate, and these images are used immediately.

The growth of capacity of communication channels and data storage devices currently not keep pace with the growth of hyperspectral data sets, so the only possible practical solution is the use the images compression [11-12]. The problem is compounded by the fact that hyperspectral data are often 16-bit, with the result that most of the popular implementations of the compression methods are not applicable.

One of the most perspective methods of hyperspectral image compression is the method [13-14], based on a hierarchical grid interpolation (HGI), which uses hierarchical image decimation and interpolation of pixels of more decimated image based on pixels of less decimated image. Advantages of this method:

1. The high efficiency at a low computational complexity. 
2. Access speed to image fragments is independent of the required resolution due to the hierarchical representation of the compressed data.

3. The possibility of strict error control [15], including mean square error and maximum error, which is especially important when processing the unique hyperspectral data (including the possibility of compression without error).

4. The ability to stabilize the rate of compressed data flow, particularly relevant for real-time systems, including on-board compression systems.

5. Possibility of using of spectral bands relationships, allowing the use of extremely high correlation of the spectral components to improve compression efficiency.

6. The possibility of compression of 16-bit spectrometers data without significant loss efficiency.

One of the key stages of the HGI method is the interpolation stage, so the task of comparative research of the interpolators' effectiveness for method HGI is actual. This problem is not researched in the published literature. In this study, we perform a comparative research of different interpolation schemes for HGI method, and propose a "rank" interpolator which has not previously been used as a part of this method.

\section{Hierarchical compression}

Compression method based on HGI uses a special representation of image $F=\{f(m, n)\}$ in the form of a nonredundant quadrotree [13] from $L$ scale levels. Let $I_{l}^{+}=\left\{\left(2^{l} m, 2^{l} n\right)\right\}$ is an index set of pixels, taken with $2^{l}$ step. The index set $I_{L-1}$ of pixels of «senior» scale level of image is simply a grid with a step $2^{L-1}$. The index set $I_{l}$ of pixels of any remaining scale level $l$ number is a grid with the step $2^{l}$, from which the pixels with the step $2^{l+1}$ are excluded:

$I_{L-1}=I_{L-1}^{+}, \quad I_{l}=I_{l}^{+} \backslash I_{l+1}^{+}, \quad 0 \leq l<L$.

We can see that the index set $I=\{(m, n)\}$ of the image is covered by the sets of scale levels indexes, and this representation is nonredundant:

$I=\bigcup_{l=0}^{L-1} I_{l}, \quad I_{j} \cap I_{k}=\varnothing \quad \forall k \neq j$.

When compression the scale levels are processed sequentially, starting with the «senior» level $(L-1)$ number. The proportion of «senior» level pixels in the total data size is negligibly small, so any trivial algorithm can be used to compress it. Common diagram of compression of any «non-senior» scale level number $l$ is shown in Fig. 1 and includes the following steps.

1) Pixels interpolation. An interpolated value is calculated for each pixel $f(m, n)$ of scale level number $l$ on the basis of already processed pixels $\bar{f}(m, n)$ : 


$$
\hat{f}(m, n)=P\left(\left\{\bar{f}(j, k),(j, k) \in I_{l+1}^{+}\right\}\right),(m, n) \in I_{l} .
$$

Interpolation is based on recovery, rather than the original pixels values in order to ensure the identity of the interpolated values for compression and decompression.

2) Calculation of difference signal (post interpolation residues):

$$
\delta(m, n)=f(m, n)-\hat{f}(m, n),(m, n) \in I_{l} .
$$

This operation reduces the signal correlation; as a result the efficiency of compression is improved

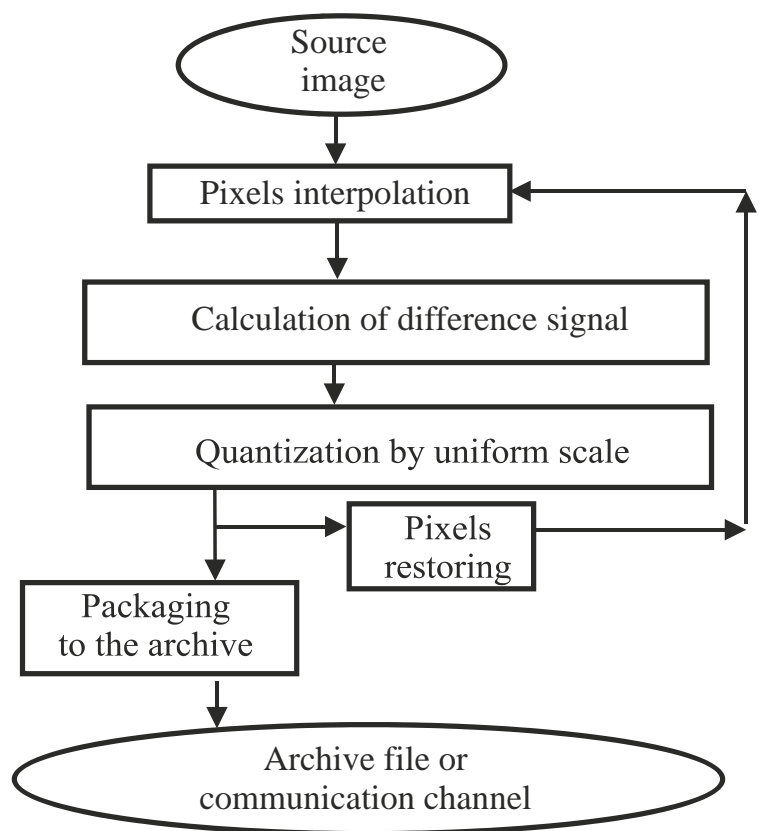

Fig. 1. The scheme of compression of any «non-senior» scale level of image by HGI method

3) Quantization of difference signal by uniform scale:

$\bar{\delta}(m, n)=\left[\frac{|\delta(m, n)|+\varepsilon_{\max }}{2 \varepsilon_{\max }+1}\right] \operatorname{sign}(\delta(m, n)),(m, n) \in I_{l}$,

where $[. .$.$] is the symbol of integer part calculation. This quantization provides the$ control [16] of maximum error $\varepsilon_{\max }$ :

$\varepsilon_{\max }=|f(m, n)-\bar{f}(m, n)|$. 
4) Packaging of quantized signal $\bar{\delta}(m, n),(m, n) \in I_{l}$ to the archive file or communication channel.

5) Pixels restoring:

$$
\bar{f}(m, n)=\hat{f}(m, n)+\bar{\delta}(m, n)\left(2 \varepsilon_{\max }+1\right),(m, n) \in I_{l} .
$$

These recovered values are used for interpolation during compression of the next scale level number $(l-1)$. In this description of the compression procedure is completed.

When decompressing the quantized signal $\bar{\delta}(m, n),(m, n) \in I_{l}$ is extracted from the archive file (or link) and unpacked. Next, reconstruction and interpolation steps identical respective compression steps are performed.

\section{Interpolation for hierarchical compression}

For interpolation in HGI method the simple schemes [17] are usually used, based on averaging the nearest already processed pixels of more decimated scale levels of the image. These schemes are differed from each other in the number of referenced pixels and interpolations sequence.

Formulas for a description of these schemes are cumbersome, but these formulas are clear from Fig. 2, so there are only formulas for the interpolator is shown in Fig. 2c:

$$
\begin{aligned}
& 4 \hat{f}\left(2^{l}(2 m+1), 2^{l}(2 n+1)\right)=f\left(2^{l+1}(m+1), 2^{l+1}(n+1)\right) \\
& +f\left(2^{l+1}(m+1), 2^{l+1} n\right)+f\left(2^{l+1} m, 2^{l+1}(n+1)\right)+f\left(2^{l+1} m, 2^{l+1} n\right), \\
& 4 \hat{f}\left(2^{l+1} m, 2^{l}(2 n+1)\right)=f\left(2^{l+1} m, 2^{l+1}(n+1)\right) \\
& +f\left(2^{l+1} m, 2^{l+1} n\right)+f\left(2^{l+1}(m-1), 2^{l}(2 n+1)\right)+f\left(2^{l+1}(m+1), 2^{l}(2 n+1)\right), \\
& 4 \hat{f}\left(2^{l}(m+1), 2^{l+1} n\right)=f\left(2^{l+1}(m+1), 2^{l+1} n\right) \\
& +f\left(2^{l+1} m, 2^{l+1} n\right)+f\left(2^{l}(2 m+1), 2^{l+1}(n-1)\right)+f\left(2^{l}(2 m+1), 2^{l+1}(n+1)\right) .
\end{aligned}
$$

Also listed in Fig. 2 interpolators in this paper we propose to use as "rank" interpolator within a hierarchical compression. Location of the reference pixels and sequence of interpolations are used in it are the same as that of the interpolator "Two crosses" (see Fig. 2c). Actually interpolation algorithm consists in constructing the ordered sample [6] of reference pixels, dropping his two extreme elements and averaging the remaining. Described truncation of ordered sample reduces the effect of noise which usually falls on the edge of ordered sample and discarded. 


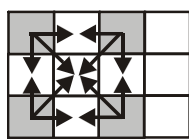

(a)

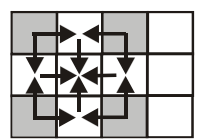

(b)

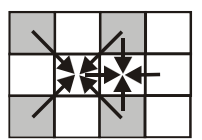

(c)

Fig. 2. Location of reference pixels and the processing sequence for the interpolators:

(a) «Diagonal cross», (b) «Straight cross», (c) «Two crosses»

\section{Experimental researches}

All of the algorithms were implemented in software by author. The developed software was used to perform the computational experiments on the series of real images of 16-bit hyperspectrometers SpecTIR [18] and AVIRIS [19]. Fragments of the spectral bands of hyperspectral image are shown in Fig. 3 (we specially selected examples of very different components of weak and strong noise). Charts of the relationship between the compression ratio and compression error are shows in Fig. 4-5. Based on thise results, the following conclusions were reached:

1. Interpolators "two crosses" and "rank" showed the greatest efficiency in compression of hyperspectral images by HGI. Interpolators "diagonal cross" and "straight cross" significantly lose.

2. Linear interpolator "two crosses" and the non-linear "rank" interpolator showed almost identical results. Consequently, the key characteristics of the interpolator are the location of the reference pixels and the sequence of interpolations, not the kind of interpolation function.

3. "Ranked" interpolator proposed in this paper for use in a hierarchical compression, showed results close to the best. This leads to the conclusion about the prospects of non-linear interpolation scheme for the HGI method.
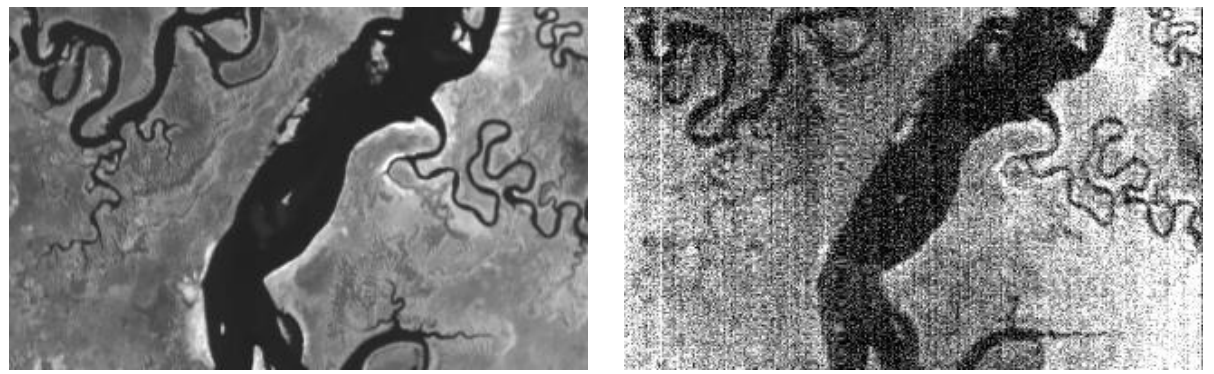

Fig. 3. The fragments of spectral bands №70, №119 of image «Gulf of Mexico Wetland Sample» of hyperspectrometer SpecTIR

\section{Conclusion}

Comparative research of different interpolation schemes for hyperspectral images compression is carried out in this paper. As a method of hyperspectral images compression we consider HGI method. Researches the effectiveness was carried out on 
the real images of the 16-bit hyperspectrometers. The dependence the compression ratio of the compression error is obtained when using different interpolators. Comparison of the three averaging interpolation schemes, as well as proposed for use in the method of the ISI «rank» scheme is performed. Key features of interpolators that affect the efficiency of compression are revealed.

In the future, we plan to develop the non-linear interpolation scheme, showing its effectiveness in a hierarchical compression of hyperspectral images. Also the adaptive selection of different interpolation schemes for different spectral components is perspective, as the characteristics of these components are substantially different.

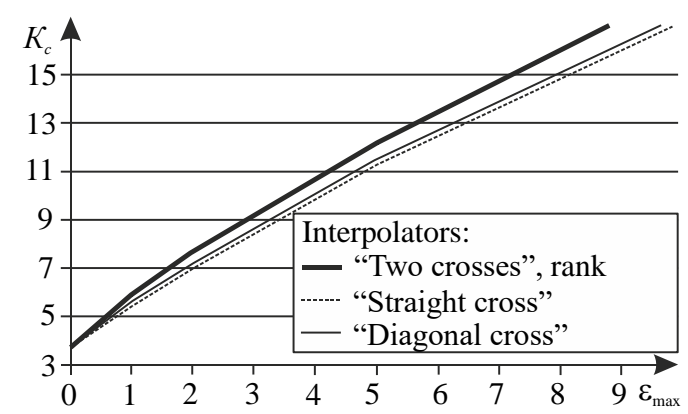

Fig. 4. Relationship between the compression ratio $K_{c}$ and maximum error $\varepsilon_{\max }$ for HGI method when using different interpolators

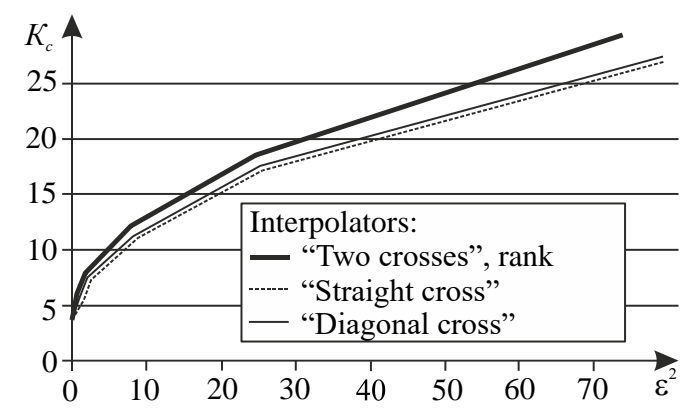

Fig. 5. Relationship between the compression ratio $K_{c}$ and square error $\varepsilon^{2}$ for HGI method when using different interpolators

\section{Acknowledgements}

This work was financially supported by the Russian Scientific Foundation (RSF), grant no. 14-31-00014 "Establishment of a Laboratory of Advanced Technology for Earth Remote Sensing". 


\section{References}

1. Chang C. Hyperspectral Data Processing: Algorithm Design and Analysis. Wiley Press, 2013; 1164 p.

2. Chang C. Hyperspectral data exploitation: theory and applications. Wiley-Interscience, 2007; $440 \mathrm{p}$.

3. Borengasser M, Hungate W, Watkins R. Hyperspectral Remote Sensing - Principles and Applications. CRC Press, 2004; 128 p.

4. Benz U, Hofmann P, Willhauck G, Lingenfelder I, Heynen M. Multi-resolution, objectoriented fuzzy analysis of remote sensing data for GIS-ready information. ISPRS Journal of Photogrammetry and Remote Sensing, 2004; 58(3): 239-258.

5. Chang $\mathrm{C}$, Chiang S. Anomaly detection and classification for hyperspectral imagery. IEEE Transactions on Geoscience and Remote Sensing, 2002; 40(6): 1314-1325.

6. Chernov AV, Chernov VM, Chicheva MA, Fursov VA, Gashnikov MV, Glumov NI, Ilyasova NY, Khramov AG, Korepanov AO, Kupriyanov AV, Myasnikov EV, Myasnikov VV, Popov SB, Sergeyev VV, Soifer VA. Computer Image Processing, Part II: Methods and algorithms. Edited by V.A. Soifer .VDM Verlag, 2010; 584 p.

7. Gashnikov MV, Glumov NI. Hyperspectral images repository using a hierarchical compression. Posters Proceedings of 23-rd International Conference on Computer Graphics, Visualization and Computer Vision (WSCG), 2015; 1-4.

8. Anderson J, Hardy E, Roach J, Witme R. A land use and land cover classification system for use with remote sensor data. US Government Printing Office, 1976; 964 p.

9. Chang C. Hyperspectral imaging: techniques for spectral detection and classification. Springer, 2003; $372 \mathrm{p}$.

10. Gashnikov MV, Glumov NI, Myasnikov VV, Chernov AV, Ivanova EV. Regional Geographic Information Systems for Gas Network Monitoring. Pattern Recognition and Image Analysis, 2015; 25(3): 418-422. DOI: 10.1134/S1054661815030062.

11. Salomon D. Data Compression. The Complete Reference. Springer-Verlag, 4ed, 2007; $1118 \mathrm{p}$.

12. Woods E, Gonzalez R. Digital Image Processing. Prentice Hall, 3ed, 2007; 976 p.

13. Gashnikov MV, Glumov NI. Hierarchical GRID Interpolation under Hyperspectral Images Compression. Optical Memory and Neural Networks (Information Optics), 2014; 23(4): 246-253.

14. Gashnikov MV, Glumov NI. Hierarchical compression for hyperspectral image storage. Computer Optics, 2014; 38(3): 482-488. [in Russian].

15. Lin S, Costello D. Error Control Coding: Fundamentals and Applications, 2ed. New Jersey: Prentice-Hall, inc. Englewood Cliffs, 2004; 1260 p.

16. Pratt W. Digital image processing. Wiley, 4ed, 2007; 807 p.

17. Gashnikov MV, Glumov NI. Hierarchical grid interpolation for hyperspectral image compression. Computer Optics, 2014; 38(1): 87-93. [in Russian].

18. SpecTIR Data - Advanced Hyperspectral and Geospatial Solutions. Corporate Headquarters SpecTIR Remote Sensing Division. URL: http://www.spectir.com/free-data-samples.

19. AVIRIS Data - Ordering Free AVIRIS Standard Data Products. Jet Propulsion Laboratory. URL: http://aviris.jpl.nasa.gov/data/free_data.html 